\title{
Puntos de corte del perímetro de la cintura para identificar sujetos con resistencia a la insulina en una población colombiana
}

\section{Cut points of waist circumference to identify subjects with insulin resistance in a colombian population}

\author{
Jaime Alberto Gallo, Juan Eugenio Ochoa, Jhon Kepa Balparda, \\ Dagnovar Aristizábal • Medellín (Colombia)
}

\section{Resumen}

Introducción: el síndrome metabólico (SM) es una condición clínica que aumenta el riesgo de enfermedad cardiovascular y se relaciona con resistencia a la insulina (RI). Aunque los consensos establecen la necesidad de puntos de corte específicos del perímetro de la cintura (PC) para cada país, con el fin de identificar sujetos con SM, en Colombia no han sido definidos.

Objetivo: definir los puntos de corte del PC en hombres y en mujeres que mejor discriminan la presencia de RI, establecer la prevalencia de SM y verificar la relación entre el PC y RI.

Material y métodos: en el contexto del estudio Diagnóstico del Riesgo Cardiovascular Global, Medellín 2007-2008, se realizó una evaluación clínica, antropométrica y de laboratorio. Con el índice de resistencia a la insulina HOMA (IR-HOMA) se definió la presencia de RI a partir del percentil 75. Se construyeron curvas de las características operativas del receptor (COR), se obtuvo el área bajo la curva (AUC) para cada sexo y se usó el índice de Youden para establecer el PC que mejor discriminaba la presencia de RI.

Resultados: se incluyeron 800 sujetos, de los cuales $44.8 \%$ fueron hombres, con un promedio de edad de 50.3 \pm 12.1 e índice de masa corporal (IMC) de $26.1 \pm 4.7$. Los sujetos con RI tuvieron mayor PC, triglicéridos y presión arterial, además, menor colesterol HDL que aquellos sin RI. Los valores que mejor discriminan la presencia de RI fueron $92 \mathrm{~cm}$ en hombres (sensibilidad 82.28\%; especificidad 70.14\%) y $84 \mathrm{~cm}$ en mujeres (sensibilidad 78.15\%; especificidad 73.98\%) (índice de Youden de 0.52 en ambos sexos). Para los hombres y mujeres el AUC fue 0.828 (IC 95\% 0.780-0.876) y 0.815 (IC 95\% 0.770-0.859), respectivamente, valor de $p<0.001$. Se encontró correlación entre el PC e IR-HOMA ( $\rho=0.65$ en los hombres y $\rho=0.62$ en las mujeres) y una prevalencia del SM del $44.9 \%$.

Conclusión: los valores de PC que mejor discriminaron la presencia de RI son $92 \mathrm{~cm}$ para hombres y $84 \mathrm{~cm}$ para mujeres. Este criterio podría ser utilizado para identificar sujetos con SM a nivel poblacional. (Acta Med Colomb 2013; 38: 118-126).

Palabras clave: síndrome metabólico, perímetro de la cintura, resistencia a la insulina, índice HOMA, factores de riesgo cardiovascular.

\footnotetext{
Abstract

Introduction: the metabolic syndrome (MS) is a clinical condition that increases the risk of cardiovascular disease and is associated with insulin resistance (IR). Although consensus establish the need of specific cut points of waist circumference (WC) for each country in order to identify subjects with MS, these have not been defined in Colombia.

Objective: to define the WC cut points in men and women that best discriminate the presence of IR, to establish the prevalence of MS and verify the relation between WC and IR.

Material and methods: in the context of the Diagnosis of the Global Cardiovascular Risk study, Medellin 2007-2008, we conducted an anthropometric and laboratory clinical evaluation. With the HOMA insulin resistance (HOMA-IR) index, we defined the presence of IR starting from the 75th percentile. Curves of the receiver operating characteristics (ROC) were made and the area under the
}

Dr. Jaime Alberto Gallo Villegas: Especialista en Medicina Aplicada a la Actividad Física y el Deporte, Magíster en Epidemiología. Centro Clínico y de Investigación SICOR. Facultad de Medicina, Universidad de Antioquia; Dr. Juan Eugenio Ochoa Múnera: Médico, Estudiante de Doctorado en Hipertensión Arterial. Universidad de Milano-Bicocca, Milán, Italia; Dr. Jhon Kepa Balparda Arias: Estudiante de Maestría en Ciencias Básicas Biomédicas. Centro Clínico y de Investigación SICOR. Corporación para Investigaciones Biológicas (CIB); Dr. Dagnovar Aristizábal Ocampo: Especialista en Medicina Interna, Cardiología y Ecocardiografía. Centro Clínico y de Investigación SICOR. Medellín (Colombia). Correspondencia: Dr. Jaime Alberto Gallo Villegas, Medellín (Colombia).

E-mail: jagallo2000@yahoo.com Recibido: 5/IX/2012 Aceptado: 27VI/2013 
curve (AUC) for each sex was obtained and the Youden index was used to establish the WC that best discriminated the presence of IR.

Results: 800 subjects were included, of whom $44.8 \%$ were male, with a mean age of $50.3 \pm 12.1$ years and body mass index $(\mathrm{BMI})$ of $26.1 \pm 4.7$. Subjects with IR had higher WC, triglycerides and blood pressure and also lower HDL cholesterol than those without IR. The values that best discriminate the presence of IR were $92 \mathrm{~cm}$ in men (sensitivity $82.28 \%$, specificity $70.14 \%$ ) and $84 \mathrm{~cm}$ in women (sensitivity 78.15\%, specificity 73.98\%) (Youden index of 0,52 in both sexes). For men and women, the AUC was 0.828 (95\% CI 0.780-0.876) and 0.815 (95\% CI 0.770 to 0.859 ), respectively, $\mathrm{p}<0.001$. A correlation between the WC and IR-HOMA ( $\rho=0.65$ in men and $\rho=0.62$ in women) as well as MS prevalence of $44.9 \%$ was found.

Conclusion: WC values that best discriminated the presence of IR are $92 \mathrm{~cm}$ for men and $84 \mathrm{~cm}$ for women. This criterion could be used to identify subjects with MS at the population level. (Acta Med Colomb 2013; 38: 118-126).

Keywords: metabolic syndrome, waist circumference, insulin resistance, HOMA index, cardiovascular risk factors.

\section{Introducción}

El síndrome metabólico (SM) es una condición clínica de alta prevalencia, caracterizada por una asociación de alteraciones metabólicas y cardiovasculares que predispone a un mayor riesgo de eventos coronarios y vasculares, a corto, mediano y largo plazo (1). El SM se asocia con resistencia a la insulina (RI) y obesidad abdominal (2) e incluye una serie de alteraciones vasculares y metabólicas, tales como hipertensión arterial (HTA), acumulación de grasa visceral, dislipidemia, intolerancia a la glucosa, diabetes mellitus (DM), y un incremento persistente de diversos factores protrombóticos, antifibrinolíticos e inflamatorios $(3,4)$.

La prevalencia del SM está en aumento, debido principalmente a cambios en el estilo de vida occidental. En el mundo se ha descrito una prevalencia del SM entre 15-30\%, particularmente en Latinoamérica la cifra reportada es del $24.9 \%$ (5). La identificación oportuna y diagnóstico adecuado de las personas con SM es de relevancia clínica, debido a que presentan un mayor riesgo de eventos cardiovasculares adversos; por tal razón, requieren de estrategias agresivas de tratamiento farmacológico y no farmacológico (6).

Diferentes organizaciones mundiales han postulado los criterios diagnósticos para detectar la presencia de SM, lo cual ha llevado a diferencias en la prevalencias reportadas en las mismas poblaciones $(7,8)$. El primer esfuerzo de esta naturaleza fue hecho por la Organización Mundial de la Salud (OMS) en 1998, quien hizo énfasis entre otros, en el rol central que tiene la RI en el desarrollo del SM. Sin embargo, debido a la dificultad técnica que propone la medición directa de la RI en la práctica diaria, los autores consideraron como aceptables las evidencias indirectas de la misma, como son la intolerancia a la glucosa y la presencia de DM tipo 2. En 2006, la Federación Internacional de Diabetes propuso a la obesidad abdominal como un prerrequisito para el diagnóstico del SM, aunque esta condición ha sido reevaluada en el último consenso (9).
La obesidad abdominal, como criterio de SM, plantea la acumulación de grasa visceral, como un marcador sensible de RI en diversas poblaciones estudiadas. Por tal razón el perímetro de la cintura (PC) ha sido propuesto como una medida antropométrica sencilla y reproducible para evaluar la presencia de RI (10). Debido a lo anterior, se han descrito diversos valores arbitrarios por encima de los cuales se debe considerar que el sujeto presenta obesidad abdominal cuando hace parte de un determinado grupo étnico.

Sin embargo, a la fecha, las distintas asociaciones internacionales insisten en la necesidad que tiene cada país o población de definir sus propios puntos de corte para establecer la presencia de obesidad abdominal, debido a las diferencias genotípicas y fenotípicas.

Según el conocimiento de los autores, en población latinoamericana, no se han realizado estudios poblacionales con el fin de identificar los valores del PC que discriminan la presencia de RI en hombres y mujeres. Por tal razón, el objetivo del presente estudio fue definir los puntos de corte del PC, tanto para hombres como para mujeres, que mejor discriminan la presencia de RI, establecer la prevalencia de SM y verificar la relación entre RI y PC en una población de sujetos mayores de 30 años, pertenecientes a una población latinoamericana en Colombia.

\section{Población de estudio}

\section{Material y métodos}

Entre junio de 2007 y junio de 2008, se realizó el Estudio Diagnóstico del Riesgo Cardiovascular Global en Medellín, que utilizó un diseño metodológico transversal, con el fin de conocer la frecuencia de los factores de riesgo mayores para la enfermedad cardiovascular, en el cual se incluyeron 800 personas, con edades entre los 30 y los 65 años. Los resultados primarios de este estudio fueron publicados por la Alcaldía de Medellín en su página web.

El tamaño de muestra para el estudio poblacional descriptivo fue calculado con base en la fórmula para la 
estimación de una proporción poblacional con un nivel de confianza de $95 \%$, una precisión de 5\% y una prevalencia estimada para HTA de $18.2 \%$. Se realizó la corrección por efecto del diseño de 3.5, que garantizó tener un tamaño de muestra suficiente para el análisis por tres categorías socioeconómicas. Así, el tamaño de muestra se estimó en 800 individuos, los cuales se consideraron como las unidades de análisis en el estudio (606 pertenecientes a las comunas y 194 pertenecientes a los corregimientos).

Para el área urbana el diseño muestral aplicado fue el muestreo probabilístico, estratificado por estrato socioeconómico y sexo, por conglomerados, trietápico y aleatorio, el cual se realizó de la siguiente forma: a partir del marco muestral de las 10145 manzanas que conforman la ciudad, se seleccionaron aleatoriamente manzanas, según su estrato socioeconómico, conformando dichas manzanas la unidad primaria de muestreo (UPM) y a su vez la primera etapa de selección.

Dentro de cada manzana seleccionada se realizó la escogencia de 10 hogares; en los mismos se seleccionaron dos personas mayores de 30 años: un hombre y una mujer, preferiblemente. Los hogares constituyeron la segunda etapa de selección y la unidad secundaria de muestreo.

Para los corregimientos (San Antonio de Prado, Belén Altavista, Santa Elena, San Cristóbal y Palmitas), se aplicó el mismo diseño muestral que para el área ubicada en las comunas, el cual se realizó de la siguiente forma: a partir del marco muestral de los 38129 predios que los conforman, se seleccionaron aleatoriamente predios, conformando dichos predios la UPM y a su vez la primera etapa de selección.

De cada uno de los corregimientos se seleccionaron siete predios, posteriormente se escogieron 41 personas mayores de 30 años de cada uno de los corregimientos. A los hogares seleccionados tanto en las comunas como en los corregimientos, se les hizo llegar una comunicación escrita en la cual, se solicitó la participación de las dos personas seleccionadas.

La selección de las personas constituyó la tercera etapa de selección y a su vez, las unidades finales de análisis de este estudio. La selección de los hogares y la invitación a la comunidad para participar en el estudio fue realizada por personal con experiencia en trabajo comunitario y cartografía.

Se incluyeron en el estudio todos los hombres y mujeres mayores de 30 años residentes en la ciudad de Medellín, elegidos aleatoriamente, que aceptaron en forma voluntaria participar en el estudio, firmaron el consentimiento informado y asistieron a los sitios seleccionados para la realización de la evaluación.

De cada individuo que participó en esta investigación se obtuvo el consentimiento informado. Se promulgó el respeto, la justicia y la beneficencia a las personas de acuerdo con las normas científicas, técnicas y administrativas para la investigación en salud del Ministerio de la Protección Social de Colombia en la Resolución 008430 de 1993, además, se tuvieron en cuenta los principios de la Declaración de
Helsinki en su última revisión $(11,12)$. El protocolo de investigación fue aprobado por el comité de ética de la Universidad CES, Medellín, Colombia.

Se excluyeron las personas con limitaciones osteomusculares que impidieran su movilización, aquellas que se encontraban en estado de embarazo, quienes tuvieran retardo cognitivo o presentaran cualquier anomalía del equilibrio emocional manifestada por comportamiento mal adaptado y funcionamiento anormal que impidiera la firma autónoma del consentimiento informado o el diligenciamiento del cuestionario.

Luego del aval del comité de ética institucional, los sujetos a estudiar fueron incluidos y evaluados durante un periodo de 12 meses. A cada sujeto se le aplicó un cuestionario estandarizado basado en el estudio INTERHEART (13), el cual incluía información sobre factores demográficos, estado socioeconómico (educación, salario), estilos de vida (tabaquismo, actividad física, patrones dietarios), uso de medicamentos, historia familiar y personal de patología cardiovascular, y factores de riesgo (HTA, DM, dislipidemia). También a cada individuo, se le realizó un examen físico, la toma de medidas antropométricas y el análisis bioquímico de una muestra de sangre en ayunas.

La aplicación del cuestionario, examen físico, la toma de medidas antropométricas y de sangre en ayunas fue realizada por un equipo, liderado por cuatro médicos miembros del equipo de investigadores, dos enfermeras con experiencia en este tipo de estudios, tres estudiantes de medicina y dos estudiantes de enfermería. Previo al inicio de la recolección de la información se llevó a cabo la capacitación, se hizo una prueba piloto y estandarización de los miembros del equipo encargados de la recolección de la información.

\section{Mediciones antropométricas}

La estatura del paciente fue medida y aproximada al $0.1 \mathrm{~cm}$ más cercano, mediante la utilización de un estadiómetro. El peso fue medido y aproximado al $0.1 \mathrm{~kg}$ más cercano, utilizando básculas calibradas. El índice de masa corporal (IMC) fue calculado al dividir el peso en kilogramos sobre el cuadrado de la estatura y expresado en $\mathrm{Kg} / \mathrm{m}^{2}$. El PC se midió con una cinta métrica estándar, sin ropa, en el abdomen, en el sitio más estrecho entre el reborde costal y la cresta iliaca. Todas las mediciones antropométricas fueron realizadas por la misma persona siguiendo un protocolo estandarizado y teniendo en cuenta las recomendaciones técnicas propuestas por la International Society for Advancement in Kinanthropometry (ISAK) - Sociedad Internacional para el Avance en Cineantropometría (14).

\section{Medición de la presión arterial}

La presión arterial, expresada en $\mathrm{mmHg}$, se definió como el valor promedio obtenido de dos tomas separadas por más de cinco minutos, y tomadas por dos médicos entrenados, siguiendo las guías de la Sociedad Europea de Hipertensión (ESH, por sus siglas en inglés) (15).Todas las mediciones 
fueron tomadas en el brazo izquierdo, al nivel del corazón, y luego de cinco minutos de reposo, mediante la utilización de un esfingomanómetro de mercurio estándar.

\section{Pruebas bioquímicas}

A todos los pacientes se les tomó una muestra de sangre venosa de $40 \mathrm{~mL}$, luego de un periodo de ayuno de ocho horas, y posteriormente fueron procesadas en las primeras seis horas luego de haber sido tomadas. El nivel de glucemia fue determinado mediante el método de glucosa-oxidasa y el de insulinemia, a partir de la técnica de inmunoensayo enzimático, siguiendo los procedimientos estandarizados del laboratorio clínico de referencia. Igualmente, se determinaron los valores de colesterol total, colesterol HDL (lipoproteína de alta densidad) y triglicéridos. El colesterol LDL (lipoproteína de baja densidad) fue calculado según la fórmula de Friedewald (16) siempre y cuando los triglicéridos estuvieran por debajo de $400 \mathrm{mg} / \mathrm{dL}$. Si el valor de los triglicéridos fue mayor que $400 \mathrm{mg} / \mathrm{dL}$, el colesterol LDL se midió en forma directa.

\section{Definición de resistencia a la insulina y síndrome metabólico}

La RI fue evaluada mediante el índice de resistencia a la insulina HOMA (IR-HOMA, Homeostasis Model Assesment of Insulin Resistance, por sus siglas en inglés), utilizando la fórmula ((glucemia $(\mathrm{mg} / \mathrm{dL}) / 18)$ x insulinemia $(\mathrm{uU} / \mathrm{mL}) / 22.5)$ como ha sido descrito previamente (17). Se definió la presencia de RI un valor del IR-HOMA por encima del percentil 75 (>2.25) según la recomendación de la Organización Mundial de la Salud.

Se utilizó la definición de SM según la presencia de tres de los cinco criterios propuestos por el último consenso intersociedades (9): glucemia en ayunas mayor de $100 \mathrm{mg} / \mathrm{dL}$ o tratamiento farmacológico para DM previamente diagnosticada; valores de presión arterial elevados $(\geq 130 \mathrm{mmHg}$ y/o $\geq 85 \mathrm{mmHg}$ para presión arterial sistólica y diastólica, respectivamente), o manejo actual para una HTA previamente diagnosticada; triglicéridos elevados en suero ( $\geq 150 \mathrm{mg} / \mathrm{dL}$ ) o tratamiento actual para una hipertrigliceridemia previamente diagnosticada; colesterol HDL disminuido $(<40 \mathrm{mg} / \mathrm{dL}$ en hombres o $<50 \mathrm{mg} / \mathrm{dL}$ en mujeres) o tratamiento actual para un colesterol HDL disminuido previamente diagnosticado; un PC aumentado según los resultados obtenidos para hombres y mujeres en el presente estudio. Siguiendo las recomendaciones más recientes, no se consideró que la presencia de obesidad abdominal fuese un criterio necesario para el diagnóstico de SM.

El diagnóstico de DM fue considerado en individuos que tenían una glucemia en ayunas $\geq 126 \mathrm{mg} / \mathrm{dL}$, o que estaban en tratamiento actual para una DM tipo 2 previamente diagnosticada. Los pacientes con DM tipo 2 no fueron excluidos del análisis final, debido a que el tratamiento con hipoglucemiantes orales disminuye la glucemia pero no afecta la RI en sujetos con DM tipo 2 no insulino-requiriente (18).

\section{Análisis estadístico}

Todos los datos cuantitativos se expresan como medias \pm desviación estándar, y los cualitativos como porcentajes. Para establecer si las variables cuantitativas provenían de una población con distribución normal se utilizó la prueba de Kolmogorov-Smirnov. La relación entre el PC y el valor del IR-HOMA fue medida mediante el coeficiente de correlación de Pearson o Spearman. Para establecer si habían diferencias entre los individuos con RI y sin ella, en las variables cuantitativas, se utilizó la prueba t de Student o U de Mann Whitney. Las diferencias en el IR-HOMA entre las categorías del PC se evaluaron con el ANOVA. Para evaluar la asociación entre las variables categóricas dicotómicas y la presencia de RI se utilizó la prueba $\chi^{2}$ de Pearson; se calcularon los respectivos OR y sus IC $95 \%$. Se utilizaron curvas de las Características Operativas del Receptor (COR) para determinar los puntos de corte del PC que mejor discriminaban la presencia de RI. Se calculó el área bajo la curva (AUC, por sus siglas en inglés) para cada sexo de manera independiente. El índice de Youden (IY), fue usado para establecer el punto de corte del PC óptimo específico por sexo, a partir de la fórmula: $I Y=$ sensibilidad + especificidad $-1=$ sensibilidad - (1-especificidad), como ha sido descrito previamente (19). Se consideró la presencia de significancia estadística un valor de $p$ inferior a 0,05 . Todos los análisis estadísticos fueron realizados mediante el software SPSS versión 15,0.

\section{Resultados}

Se incluyeron 800 sujetos, con una promedio de edad $50.3 \pm 12.1$ años, IMC de $26.1 \pm 4.7 \mathrm{Kg} / \mathrm{m}^{2}$ y un PC de $85.9 \pm$ $12.8 \mathrm{~cm}$. El $44.8 \%$ de la muestra estuvo conformada por individuos de sexo masculino. Los sujetos con RI presentaron un promedio del IR-HOMA de $4.4 \pm 3.5$, mientras aquellos sin RI el promedio fue de $1.2 \pm 0.5$.

En la Tabla 1 se observa la comparación de hallazgos entre aquellos sujetos que presentaban RI y aquellos que no, donde se resalta cómo los primeros presentaban valores más elevados de presión arterial sistólica y diastólica, PC, IMC, glucemia en ayunas, colesterol total, triglicéridos e insulinemia que aquellos sin RI. De manera similar, los individuos que presentaban RI tenían una mayor frecuencia de antecedentes personales de HTA, DM, angina, dislipidemia y de actividad física de baja y moderada intensidad.

Se encontró correlación entre el PC e IMC en toda la población de estudio $(\rho=0.785 p<0.001)$. En las Figuras 1 y 2 se observa la relación entre el PC y el IR-HOMA en los individuos incluidos en esta investigación. La Figura 1 muestra los gráficos de dispersión que representan la correlación entre el PC y el IR-HOMA en hombres y mujeres ( $\rho=0.62$ para hombres; $\rho=0.65$ para mujeres; en ambos casos $p<0.001)$. En la Figura 2 se observan los promedios del IR-HOMA y sus respectivos IC $95 \%$, a través de las diferentes categorías del PC tanto en hombres como 
Tabla 1. Comparación de los hallazgos clínicos y los antecedentes personales entre los sujetos con y sin resistencia a la insulina según el índice de resistencia a la insulina HOMA, en una muestra de 800 sujetos evaluados en el contexto del Estudio Diagnóstico del Riesgo Cardiovascular Global en Medellín 2007-2008.

\begin{tabular}{|c|c|c|c|c|c|c|}
\hline \multirow{3}{*}{ Variables } & \multicolumn{4}{|c|}{ Resistencia a la insulina } & \multirow{3}{*}{$\begin{array}{l}\text { Diferencia de Medias y OR* } \\
\text { (IC 95\%) }\end{array}$} & \multirow{3}{*}{ Valor de $p$} \\
\hline & \multicolumn{2}{|c|}{$\mathrm{Si}(n=\mathbf{2 0 0})$} & \multicolumn{2}{|c|}{ No $(n=600)$} & & \\
\hline & Media \pm & $\mathrm{DE}(\%)$ & Media \pm & DE $(\%)$ & & \\
\hline Edad (años) & 53.2 & 12.0 & 49.3 & 11.9 & $3.9(2.0-5.8)$ & $<0.001$ \\
\hline Hombres & & $39.6 \%$ & & $46.4 \%$ & $1.31(0.94-1.82)^{*}$ & 0.09 \\
\hline Presión arterial sistólica supino $(\mathrm{mm} / \mathrm{Hg})$ & 139.1 & 24.0 & 128.1 & 22.3 & $11.2(7.5-14.8)$ & $<0.001$ \\
\hline Presión arterial diastólica supino $(\mathrm{mm} / \mathrm{Hg})$ & 81.9 & 11.9 & 77.8 & 11.3 & $3.9(2.1-5.8)$ & $<0.001$ \\
\hline Perímetro abdominal (cm) & 94.0 & 10.5 & 83.2 & 12.5 & $11.3(9.7-12.9)$ & $<0.001$ \\
\hline Índice de masa corporal $\left(\mathrm{kg} / \mathrm{m}^{2}\right)$ & 29.7 & 4.5 & 25.0 & 4.2 & $4.7(4.0-5.4)$ & $<0.001$ \\
\hline Glucemia en ayunas $(\mathrm{mg} / \mathrm{dL})$ & 107.5 & 40.3 & 83.9 & 12.6 & $23.5(19-8-27.2)$ & $<0.001$ \\
\hline Colesterol total $(\mathrm{mg} / \mathrm{dL})$ & 231.4 & 48.9 & 219.1 & 45.3 & $12.1(4.7-19.6)$ & 0.001 \\
\hline Colesterol HDL (mg/dL) & 36.7 & 10.4 & 40.8 & 11.2 & $-4.2(-6.0-2.5)$ & $<0.001$ \\
\hline Triglicéridos (mg/dL) & 209.2 & 100.3 & 148.0 & 97.3 & $63.2(46.9-79.5)$ & $<0.001$ \\
\hline Colesterol LDL (mg/dL) & 152.4 & 44.3 & 148.4 & 40.4 & $4.2(-2.4-10.9)$ & 0.405 \\
\hline Insulinemia (uU/mL) & 16.8 & 9.5 & 5.6 & 2.5 & $11.1(10.3-11.9)$ & $<0.001$ \\
\hline IR-HOMA & 4.4 & 3.5 & 1.2 & 0.5 & $3.1(2.9-3.3)$ & $<0.001$ \\
\hline Antecedente personal de hipertensión & & $43.2 \%$ & & $20.1 \%$ & $3.02(2.14-4.27)^{*}$ & $<0.001$ \\
\hline Antecedente personal de diabetes & & $19.6 \%$ & & $2.2 \%$ & $10.93(5.69-20.97)^{*}$ & $<0.001$ \\
\hline Antecedente personal de angina & & $6.6 \%$ & & $2.5 \%$ & $2.74(1.28-5.86)^{*}$ & 0.007 \\
\hline Antecedente personal de infarto & & $1.5 \%$ & & $1.3 \%$ & $1.31(0.29-4.30)^{*}$ & 0.857 \\
\hline Antecedente personal de dislipidemia & & $37.9 \%$ & & $20.8 \%$ & $2.33(1.64-3.31)^{*}$ & $<0.001$ \\
\hline Actividad física baja y moderada & & $90.5 \%$ & & $81.8 \%$ & $2.10(1.25-3.53)^{*}$ & 0.004 \\
\hline
\end{tabular}

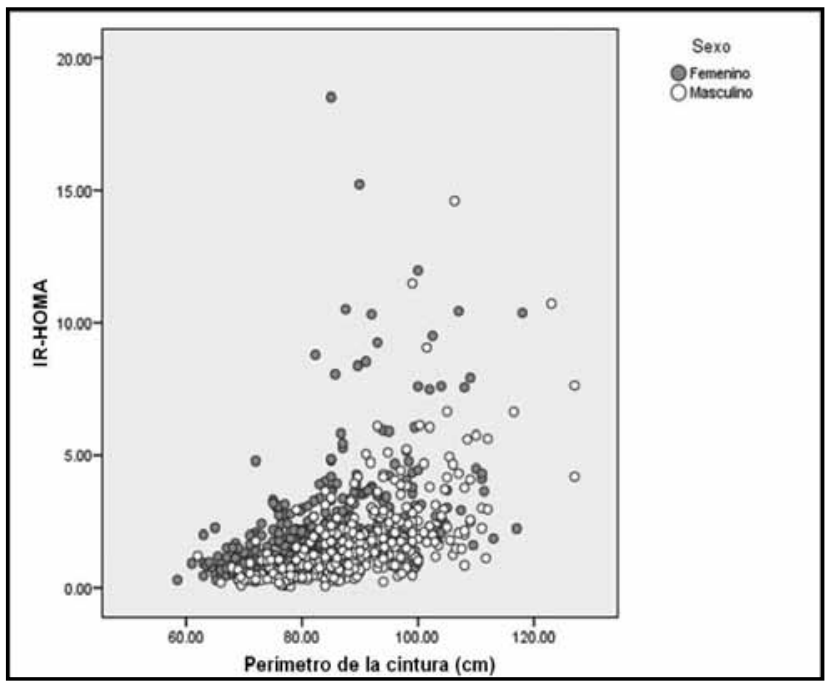

Figura 1. Relación entre el índice de resistencia a la insulina HOMA(IR-HOMA) y el perímetro de la cintura según el sexo en la muestra de 800 sujetos evaluados en el contexto del Estudio Diagnóstico del Riesgo Cardiovascular Global en Medellín 2007-2008. Coeficiente de correlación de Spearman $\rho=0,65$ para las mujeres y $\rho=0,62$ para los hombres, $p<0,001$.

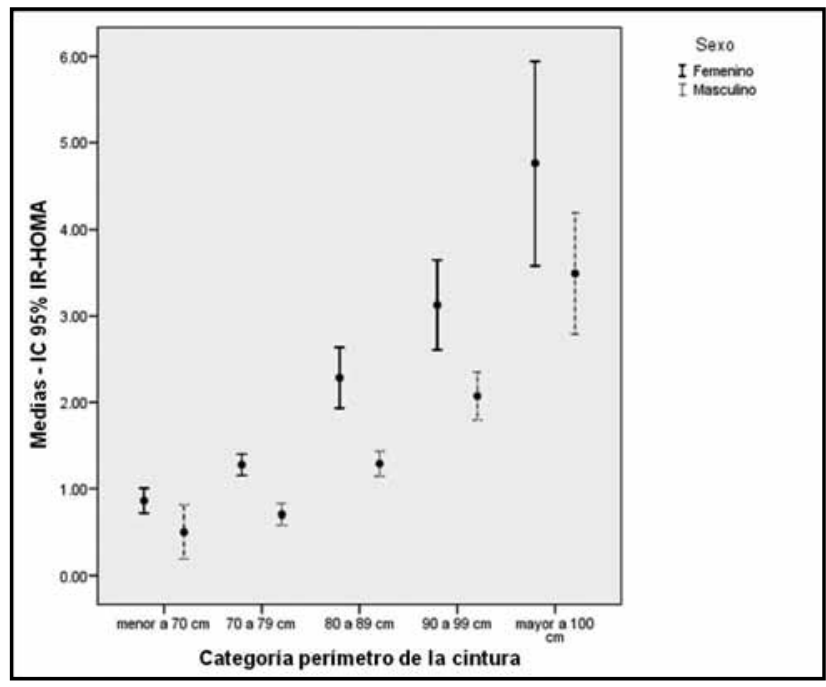

Figura 2. Medias y sus respectivos IC 95\% del índice de resistencia a la insulina HOMA (IR-HOMA), a través de las diferentes categorías del perímetro de la cintura tanto en hombres como en mujeres, en una muestra de 800 sujetos evaluados en el contexto del Estudio Diagnóstico del Riesgo Cardiovascular Global en Medellín 2007-2008 ( $p$ del ANOVA < 0,001 , en hombres y mujeres). 
en mujeres ( $p$ del ANOVA $<0.001$ en hombres y mujeres). En ambas figuras se evidencia un aumento del IR-HOMA a medida que se incrementa el PC.

Con los valores del PC e IR-HOMA, de los individuos incluidos en el estudio, se construyeron las curvas COR para hombres y mujeres como se observa en la Figura 3. Para los hombres el AUC fue 0.828 (IC 95\% 0.780-0.876), valor de $p<0.001$; y para las mujeres, el AUC fue 0.815 (IC $95 \%$ $0.770-0.859$ ) valor de $p<0.001$.

El punto de corte del PC que mejor discrimina la presencia de RI, fue $92 \mathrm{~cm}$ para los hombres (sensibilidad 82.28\%; especificidad $70.14 \%$ ) y $84 \mathrm{~cm}$ para mujeres (sensibilidad $78.15 \%$; especificidad 73.98\%), índice de Youden de 0.52 en ambos casos. La proporción de personas que cumplieron con tres o más criterios para SM fue $44.9 \%$ (hombres $43.3 \%$ y mujeres $46.2 \%)$.

\section{Discusión}

El SM es un conjunto de alteraciones metabólicas relacionadas que aumentan el riesgo de sufrir eventos coronarios adversos a corto, mediano y largo plazo (20). Su importancia radica principalmente en la alta prevalencia y los altos costos que la entidad con sus complicaciones y enfermedades asociadas significan para los sistemas de salud en todo el mundo (21).
Los resultados presentados en este artículo son producto de un estudio poblacional realizado en la ciudad de Medellín, Colombia, con miras a evaluar el estado actual de la salud cardiovascular de manera local. Se analizaron, entre otros, los valores de glucemia e insulinemia en ayunas, para construir el IR-HOMA que permitiera evidenciar la presencia de RI, tomando aquellos sujetos cuyo índice se encontró por encima del percentil 75 , como ha sido descrito previamente (22).

A partir del IR-HOMA se definió el PC, expresado en centímetros, que mejor discriminaba la presencia de RI, con el objetivo de obtener los puntos de corte con las mejores características operativas para la población local, con el fin de mejorar los procesos diagnósticos en salud cardiovascular que se lleven a cabo dentro de la región y evitar la extrapolación de datos obtenidos en otras poblaciones, los cuales pueden variar debido a las características genotípicas y fenotípicas, que señalan cambios metabólicos diferenciales según el grado de obesidad abdominal.

En estudios previos, Faerchy colaboradores demostraron que la disminución en la sensibilidad a la insulina, y la resistencia a la misma, son alteraciones subclínicas, que predisponen de manera importante a un riesgo elevado de DM (23), lo cual provocaría la necesidad de hacer una vigilancia activa de las mismas en la población general (24),
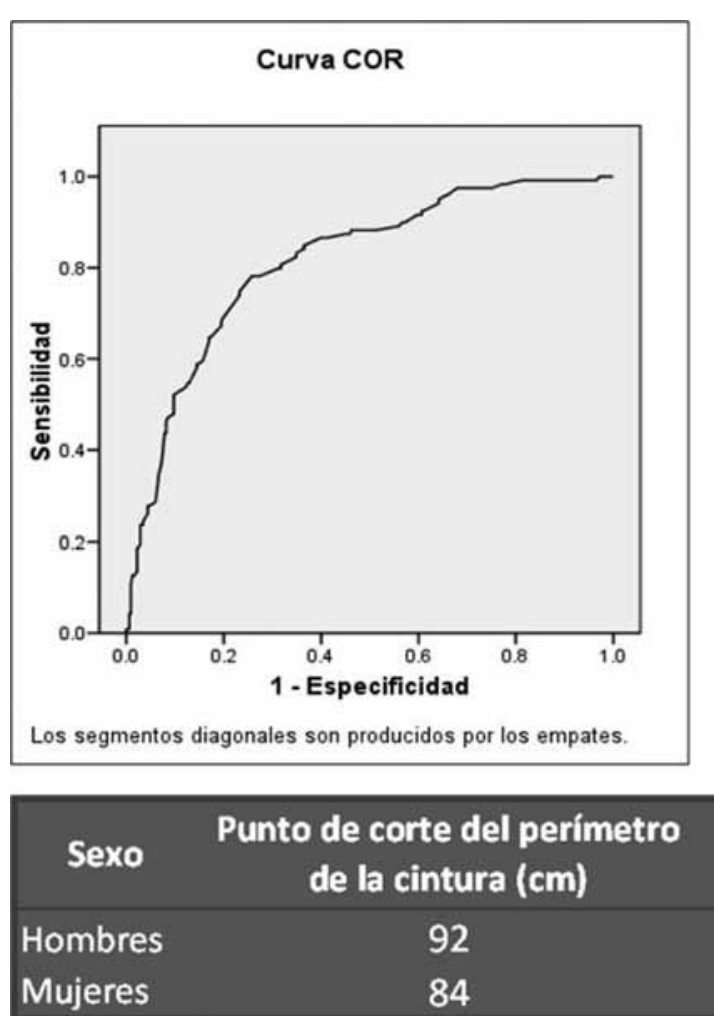

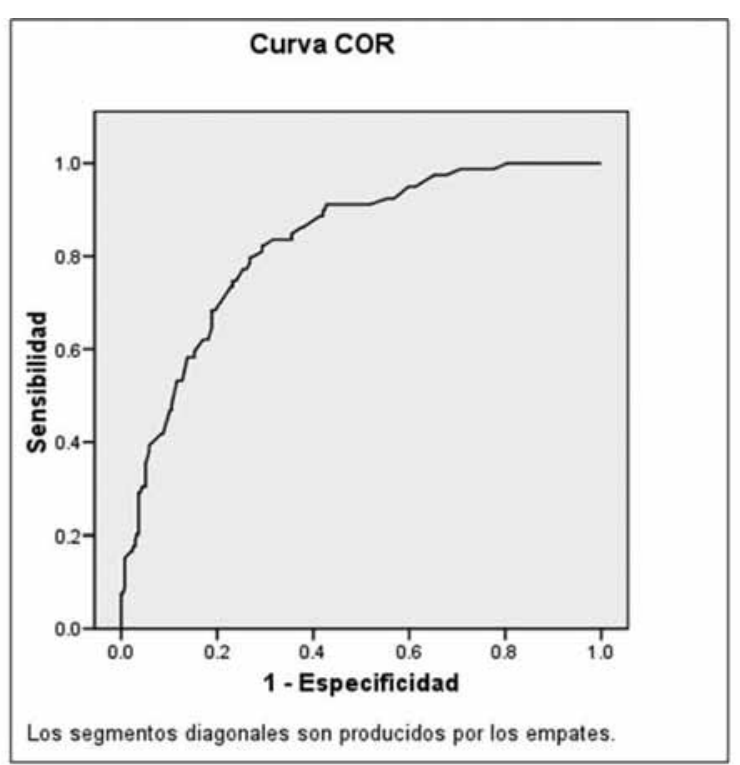

\begin{tabular}{|ccc|}
$\begin{array}{c}\text { Sensibilidad } \\
(\%)\end{array}$ & $\begin{array}{c}\text { Especificidad } \\
(\%)\end{array}$ & $\begin{array}{c}\text { Índice de } \\
\text { Youden }\end{array}$ \\
\hline 82,28 & 70,14 & 0,52 \\
78,15 & 73,98 & 0,52 \\
\hline
\end{tabular}

Figura 3. Curvas de las Características Operativas del Receptor (COR) del perímetro de la cintura según la presencia de resistencia a la insulina en hombres (izquierda) y mujeres (derecha) en una muestra de 800 sujetos evaluados en el contexto del Estudio Diagnóstico del Riesgo Cardiovascular Global en Medellín 2007-2008. Área bajo la curva (AUC) en hombres 0,828 (IC $95 \%$ 0,780-0,876), valor de $p<0,001$; y en las mujeres, 0,815 (IC 95\% 0,770-0,859) valor de $p<0,001$. 
especialmente en aquellos individuos con otros factores de riesgo cardiovascular.

También, nuestro grupo de investigación, reportó cambios desfavorables en el metabolismo de los lípidos y la glucosa, además de alteraciones hemodinámicas iniciales, como son aumento del acortamiento miocárdico y la función sistólica del ventrículo izquierdo, que sugieren una mayor estimulación cardiaca y contractilidad por activación del tono simpático en adultos jóvenes no obesos con resistencia a la insulina (25).

Sin embargo, la medición rutinaria de la insulinemia en todos los pacientes no es una estrategia viable económicamente para la mayoría de los servicios de la salud, por lo que se hace necesario el desarrollo de herramientas clínicas económicas y fáciles de realizar que se relacionen de manera adecuada con un estado alterado en la sensibilidad a la insulina.

En este punto, diversas mediciones antropométricas, como el PC, IMC, relación cintura-cadera y relación cintura-estatura $(24,26-28)$ han demostrado ser marcadores promisorios de utilidad para la estimación clínica de alteraciones en la sensibilidad a la insulina. La medición del PC es particularmente importante, por su facilidad no sólo en su medición sino en su interpretación (27). Sin embargo, como ya se mencionó anteriormente, el principal inconveniente de este tipo de mediciones antropométricas es su variabilidad en las diferentes regiones del mundo debido a componentes culturales, alimentarios y genéticos de las distintas poblaciones, lo que dificulta la extrapolación de datos de los estudios llevados a cabo en otros países. Lo anterior, genera la necesidad de disponer de valores antropométricos propios para cada población particularmente para Latinoamérica y Colombia (29).

Al comparar distintas mediciones clínicas y paraclínicas entre los sujetos con y sin RI, según el IR-HOMA, se encontró que los primeros, no sólo tenían un PC y un IMC más elevado, sino también una mayor presión arterial y alteraciones del perfil lipídico. Este hallazgo está en concordancia con el concepto ya descrito de la relación entre los distintos componentes que hacen parte del SM, en donde se observa agregación de los mismos en los pacientes, lo cual incrementa el riesgo metabólico (30). En esta investigación, se encontró que aquellos sujetos con RI tenían unos mayores valores en el PC e IMC como ha sido reportado $(26,27)$. Resulta importante el hecho que no se encontraron diferencias en las frecuencias del sexo entre los grupos con y sin RI, lo cual debe alertar al clínico sobre la necesidad de descartar este tipo de condiciones no sólo en los individuos de sexo masculino sino también en las mujeres.

Con respecto a la relación entre el PC e IR-HOMA, se observó una correlación moderada, estadísticamente significativa, entre las dos variables, lo que corrobora la evidencia existente de la asociación entre la obesidad visceral (representada indirectamente por el PC) y la presencia de alteraciones en la homeostasis glucémica. En las figuras, se muestra que el incremento en el IR-HOMA según cambia el PC sigue un patrón exponencial. A valores bajos del PC se observan mínimas elevaciones en la RI, pero luego en la medida que aumenta el PC, se alcanza un valor crítico que se asocia con mayores cambios en el IR-HOMA. Lo anterior señala la existencia de un "umbral" en el PC a partir del cual se encuentran la mayoría de los pacientes con alteraciones en la sensibilidad a la acción de la insulina, y está en concordancia con los hallazgos previos de nuestro grupo, el cual reportó el fenómeno del gradiente insulínico en una población colombiana (31).

Se puede agregar que previamente habíamos establecido el punto de corte para la presencia de RI en una muestra de población universitaria de menor edad, a partir del percentil 75 , un valor de insulinemia en ayuno mayor de $14.65 \mathrm{uU} / \mathrm{mL}$ y del IR-HOMA mayor de 3.28 (datos no publicados). El presente estudio, incluyó una muestra poblacional $(n=800)$, y teniendo en cuenta la misma definición (percentil 75), los valores serían para la insulinemia e IR-HOMA, 9.99 $\mathrm{uU} / \mathrm{mL}$ y 2.25 , respectivamente. En los individuos con RI encontramos unos valores promedio de insulinemia e IRHOMA menores que los descritos en el estudio anterior $(16.8 \pm 9.5 \mathrm{uU} / \mathrm{mL}$ vs $20.79 \pm 7.41 \mathrm{uU} / \mathrm{mL}$ y $4.4 \pm 3.5$ vs $4.95 \pm 2.33$, respectivamente) (32). Dichas diferencias pueden ser debidas a las características de las poblaciones evaluadas, factores medioambientales que pudieron haber cambiado en el tiempo y a la técnica de medición empleada. Así, basados en nuestros resultados, la presencia de un valor de insulinemia en ayuno mayor de $9.99 \mathrm{uU} / \mathrm{mL}$ o un IRHOMA mayor de 2.25 es compatible con RI y debe estar acompañado de la contraparte metabólica y antropométrica como ha sido descrito en nuestros resultados.

Los coeficientes de correlación obtenidos entre el PC e IR-HOMA en el presente estudio ( $\rho=0.62$ para hombres y $\rho=0.65$ para mujeres) se encuentran por encima de los valores reportados por autores en otras poblaciones brasileñas ( 24 , 33) y taiwanesas (34).

Con respecto a los puntos de corte del PC más apropiados para detectar RI, según las curvas COR y el índice de Youden (19), se encontró que los valores que brindan unas mejores características operativas para detectar RI son $92 \mathrm{~cm}$ para los hombres y $84 \mathrm{~cm}$ para las mujeres. Estos valores difieren en cierta medida con lo propuesto por la International Diabetes Federation (IDF) (35), quienes plantean que los puntos de corte óptimos del PC que señalan obesidad abdominal son $94 \mathrm{~cm}$ para los hombres de ascendencia europea y 80 $\mathrm{cm}$ para las mujeres de cualquier etnia, los cuales podrían considerarse como los valores que se han utilizado en los estudios colombianos y latinoamericanos que han evaluado la frecuencia del SM $(5,8,39)$. Aún más, difiere de manera importante con respecto a los valores propuestos por el Adult Treatment Panel III (ATP III) (36), para el diagnóstico clínico del SM, quienes han sugerido que se debería establecer la presencia de obesidad abdominal en los hombres cuando se encuentra un PC por encima de $102 \mathrm{~cm}$, y para 
mujeres por encima de $88 \mathrm{~cm}$. Claramente, la utilización de estos valores de referencia, sugeridos por el ATP III en la población latinoamericana, generaría un incremento en la especificidad, pero una disminución en la sensibilidad, en la definición de la presencia de RI, si se compara con los datos obtenidos en el estudio que aquí se presenta.

En una investigación, que incluyó militares colombianos, sugieren un valor de $88 \mathrm{~cm}$ del PC para hombres como un criterio de riesgo metabólico (37). En otro trabajo, que incluyó población latinoamericana, se describe un PC de $94 \mathrm{~cm}$ para los hombres y $90 \mathrm{~cm}$ para las mujeres como criterio de un exceso de tejido adiposo visceral (38). Sin embargo, en ambos estudios, la definición de los puntos de corte del PC fue a partir de variables diferentes a la RI y no son estudios poblacionales.

$\mathrm{Si}$ se tiene en cuenta que las personas con SM, o aquellas con alto riesgo para el desarrollo de DM y enfermedad cardiovascular, deberían ser diagnosticadas y manejadas de forma temprana y con intervenciones precisas, se entiende que al disminuir la sensibilidad del método diagnóstico, se está dejando de tratar un número considerable de personas que en realidad sí tienen una condición clínica metabólica. Al comparar los valores de PC propuestos por la IDF con los obtenidos en este estudio, se evidencia que no son muy diferentes (sólo $2 \mathrm{~cm}$ en los hombres y $4 \mathrm{~cm}$ en las mujeres), pero se excluirían de un tratamiento más intensivo personas con un alto riesgo metabólico y cardiovascular. La incorporación de un método diagnóstico de tamización en la población, para definir la presencia de una condición clínica, se debe elegir teniendo en cuenta las características operativas, en particular una alta sensibilidad que permita detectar adecuadamente a la mayoría de los sujetos que se beneficiarían de un tratamiento.

En esta investigación se utilizó la definición más reciente de SM recomendada por el consenso intersociedades y se incluyó el punto de corte del PC descrito en nuestros resultados (9). Se encontró una prevalencia de SM del 44.9\%, la cual es alta, cuando se compara con la reportada en otros países latinoamericanos (5) y con ciudades de otras regiones de Colombia $(8,39)$. Este hallazgo puede estar relacionado con la incorporación de hábitos del estilo de vida del mundo occidental, la rápida transición epidemiológica y el incremento en la prevalencia de la obesidad en nuestros países. El estudio INTERHEART para Latinoamérica reportó la obesidad abdominal como el factor de riesgo para un primer infarto de miocardio con el más alto riesgo atribuible poblacional (40), lo cual plantea la necesidad del desarrollo de estrategias de salud pública de promoción de la salud y prevención de la enfermedad cardiovascular que tenga en cuenta los factores de riesgo modificables.

\section{Conclusión}

En este estudio, se encontró correlación entre el PC y RI. La RI se asocia con la edad, presión arterial, metabolismo de los lípidos, antecedentes de DM y enfermedad cardiovascular e inactividad física. Los valores de PC que mejor discriminaron la presencia de RI son $92 \mathrm{~cm}$ para los hombres y $84 \mathrm{~cm}$ para las mujeres. Este criterio podría ser utilizado para identificar sujetos con SM a nivel poblacional. Teniendo en cuenta la definición más reciente de SM y los valores de PC sugeridos en este estudio, se encontró una alta prevalencia de SM la cual fue del $44.9 \%$.

\section{Agradecimientos}

Los autores desean agradecer a la comunidad del municipio de Medellín que participó en el estudio, estudiantes y profesionales que participaron en la recolección de la información, a la Secretaría de Salud del municipio de Medellín, a la Clínica Medellín y a la Universidad CES.

\section{Reconocimientos}

El presente estudio ha recibido los siguientes reconocimientos:

"Mejor Trabajo de Investigación Clínica", otorgado por la Asociación Colombiana de Endocrinología, con base en presentación realizada en el 11 Congreso Colombiano de Endocrinología. Cali, Colombia, mayo de 2011.

"Mejor Trabajo Extranjero de Investigación", otorgado por la Sociedad Argentina de Medicina, con base en presentación realizada en el Congreso Internacional de Clínica Médica y Medicina Interna 2011. Buenos Aires, Argentina, noviembre de 2011.

\section{Financiación}

El presente estudio fue financiado por la Secretaria de Salud del Municipio de Medellín, la Clínica Medellín y la Universidad CES, a partir del convenio de asociación No 4700028957 de 2007.

\section{Conflictos de Interés}

Los autores no tienen ningún conflicto de interés que declarar.

\section{Referencias}

1. Tesauro M, Canale MP, Rodia G, Di Daniele N, Lauro D, Scuteri A, et al. Metabolic syndrome, chronic kidney, and cardiovascular diseases: role of adipokines. Cardiol Res Pract 2011; 2011: 653182.

2. Gallagher EJ, Leroith D, Karnieli E. Insulin resistance in obesity as the underlying cause for the metabolic syndrome. Mt Sinai J Med 2010; 77(5): 511-23.

3. Alshehri AM. Metabolic syndrome and cardiovascular risk. J Family Community Med 2010; 17(2): 73-8.

4. Nesto RW. Beyond low-density lipoprotein: addressing the atherogenic lipid triad in type 2 diabetes mellitus and the metabolic syndrome. Am J Cardiovasc Drugs 2005; 5(6): 379-87.

5. Marquez-Sandoval F, Macedo-Ojeda G, Viramontes-Horner D, Fernandez Ballart J, Salas Salvado J, Vizmanos B. The prevalence of metabolic syndrome in Latin America: a systematic review. Public Health Nutr 2011; 13: 1-12.

6. Greer DM, Hill DC. Implementing an evidence-based metabolic syndrome prevention and treatment program utilizing group visits. J Am Acad Nurse Pract 2011; 23(2): 76-83.

7. Paternina-Caicedo AJ, Alcala-Cerra G, Paillier-Gonzales J, Romero-Zarante AM, Alvis-Guzman N. [Agreement between three definitions of metabolic syndrome in hypertensive patients]. Rev Salud Publica 2009; 11(6): 898-908.

8. Mora Garcia G, Salguedo Madrid G, Ruiz Diaz M, Ramos Clason E, Alario Bello A, Fortich A, et al. [Agreement between Five Definitions of Metabolic Syndrome: Cartagena, Colombia]. Rev Esp Salud Publica 2012; 86(3): 301-11.

9. Alberti KG, Eckel RH, Grundy SM, Zimmet PZ, Cleeman JI, Donato KA, et al. Harmonizing the metabolic syndrome: a joint interim statement of the International Diabetes Federation Task Force on Epidemiology and Prevention; National Heart, Lung, and Blood Institute; American Heart Association; World Heart Federation; International Atherosclerosis Society; and International Association for the Study of Obesity. Circulation 2009; 120(16): 1640-5.

10. Park YM, Kwon HS, Lim SY, Lee JH, Yoon KH, Son HY, et al. Optimal waist circumference cutoff value reflecting insulin resistance as a diagnostic criterion of metabolic syndrome in a nondiabetic Korean population aged 40 years and over: the Chungju Metabolic Disease Cohort (CMC) study. Yonsei Med J 2010 51(4): $511-8$

11. Resolución 8430 de 1993 Bogotá: Ministerio de Protección Social; 1993.

12. Schuklenk U. Helsinki Declaration revisions. Issues Med Ethics 2001; 9(1): 29.

13. Yusuf S, Hawken S, Ounpuu S, Dans T, Avezum A, Lanas F, et al. Effect of 
potentially modifiable risk factors associated with myocardial infarction in 52 countries (the INTERHEART study): case-control study. Lancet 2004; 364(9438): 937-52.

14. Norton K, Olds T. Anthropometrica: a textbook of body measurement for sports and health courses. Sidney: University of New South Wales Press; 2004.

15. Mancia G, De Backer G, Dominiczak A, Cifkova R, Fagard R, Germano G, et al. 2007 Guidelines for the Management of Arterial Hypertension: The Task Force for the Management of Arterial Hypertension of the European Society of Hypertension (ESH) and of the European Society of Cardiology. ESC 2007; 25(6): 1105-87.

16. Hata Y, Nakajima K. Application of Friedewald's LDL-cholesterol estimation formula to serum lipids in the Japanese population. Jpn Circ J 1986; 50(12): 1191-200.

17. Matthews DR, Hosker JP, Rudenski AS, Naylor BA, Treacher DF, Turner RC. Homeostasis model assessment: insulin resistance and beta-cell function from fasting plasma glucose and insulin concentrations in man. Diabetologia $1985 ; 28(7): 412-9$

18. Emoto M, Nishizawa Y, Maekawa K, Hiura Y, Kanda H, Kawagishi T, et al. Homeostasis model assessment as a clinical index of insulin resistance in type 2 diabetic patients treated with sulfonylureas. Diabetes Care 1999; 22(5): 818-22.

19. Akobeng AK. Understanding diagnostic tests 3: Receiver operating characteristic curves. Acta Paediatr 2007; 96(5): 644-7.

20. Raimundo M, Lopes JA. Metabolic syndrome, chronic kidney disease, and cardiovascular disease: a dynamic and life-threatening triad. Cardiol Res Pract 2011; 2011: 747861.

21. Wille E, Scholze J, Alegria E, Ferri C, Langham S, Stevens W, et al. Modelling the costs of care of hypertension in patients with metabolic syndrome and its consequences, in Germany, Spain and Italy. Eur J Health Econ 2011; 12(3): 205-18

22. Burrows RA, Leiva LB, Weisstaub G, Lera LM, Albala CB, Blanco E, et al. High HOMA-IR, adjusted for puberty, relates to the metabolic syndrome in overweight and obese Chilean youths. Pediatr Diabetes 2011; 12(3 Pt 2): 212-8.

23. Faerch K, Vaag A, Holst JJ, Hansen T, Jorgensen T, Borch-Johnsen K. Natural history of insulin sensitivity and insulin secretion in the progression from normal glucose tolerance to impaired fasting glycemia and impaired glucose tolerance: the Inter99 study. Diabetes Care 2009; 32(3): 439-44.

24. Matos LN, Giorelli Gde V, Dias CB. Correlation of anthropometric indicators for identifying insulin sensitivity and resistance. Sao Paulo Med J 2011; 129(1): 30-5.

25. Gallo J, Aristizabal D, Segura A, Correa M, Zapata N. Relationship of insulin resistance with heart structure, function, and metabolism in young, nonobese adults. Acta Med Colomb 2008; 33: 117-26.

26. Kondaki K, Grammatikaki E, Pavon DJ, Manios Y, Gonzalez-Gross M, Sjostrom M, et al. Comparison of several anthropometric indices with insulin resistance proxy measures among European adolescents: The Helena Study. Eur J Pediatr 2010; 170: 731-739.
27. Lopatynski J, Mardarowicz G, Szczesniak G. A comparative evaluation of waist circumference, waist-to-hip ratio, waist-to-height ratio and body mass index as indicators of impaired glucose tolerance and as risk factors for type-2 diabetes mellitus. Ann Univ Mariae Curie Sklodowska Med 2003; 58(1): 413-9.

28. Hadaegh F, Zabetian A, Harati H,Azizi F. Waist/height ratio as a better predictor of type 2 diabetes compared to body mass index in Tehranian adult men a 3.6-year prospective study. Exp Clin Endocrinol Diabetes 2006; 114(6): 310-5.

29. Lopez-Jaramillo P, Rueda-Clausen CF, Silva FA. The utility of different definitions of metabolic syndrome in Andean population. Int J Cardiol 2007; 116(3): 421-2.

30. Deboer MD. Underdiagnosis of Metabolic Syndrome in Non-Hispanic Black Adolescents: A Call for Ethnic-Specific Criteria. Curr Cardiovasc Risk Rep 2010; 4(4): 302-10.

31. Aristizabal D, Gallo J, Fernandez R, Restrepo MA, Zapata N, Correa M. The insulin gradient phenomenon: a manifestation of the effects of body weight on blood pressure and insulin resistance. J Cardiometab Syndr 2008; 3(4): 218-23.

32. Fernandez RG, L. Gallo, E. et al. Sensibilidad a la insulina y su relación con la presión arterial y otros factores de riesgo cardiovascular. Acta Médica Colomb 1997; 22(1): 8-17.

33. Vasques AC, Rosado LE, Rosado GP, Ribeiro Rde C, Franceschini Sdo C, Geloneze B, et al. Different measurements of the sagittal abdominal diameter and waist perimeter in the prediction of HOMA-IR. Arq Bras Cardiol 2009 Nov; 93(5): 511-8.

34. Chen CN, Chuang LM, Wu YT. Clinical measures of physical fitness predict insulin resistance in people at risk for diabetes. Phys Ther 2008; 88(11): 1355-64.

35. Baik I. Optimal cutoff points of waist circumference for the criteria of abdominal obesity: comparison with the criteria of the International Diabetes Federation. Circ $J$ 2009; 73(11): 2068-75.

36. Grundy SM, Cleeman JI, Daniels SR, Donato KA, Eckel RH, Franklin BA, et al. Diagnosis and management of the metabolic syndrome. An American Heart Association/National Heart, Lung, and Blood Institute Scientific Statement. Executive summary. Cardiol Rev 2005; 13(6): 322-7.

37. Perez M, Casas JP, Cubillos-Garzon LA, Serrano NC, Silva F, Morillo CA, et al. Using waist circumference as a screening tool to identify Colombian subjects at cardiovascular risk. Eur J Cardiovasc Prev Rehabil 2003; 10(5): 328-35.

38. Aschner P, Buendia R, Brajkovich I, Gonzalez A, Figueredo R, Juarez XE, et al. Determination of the cutoff point for waist circumference that establishes the presence of abdominal obesity in Latin American men and women. Diabetes Res Clin Pract 2011; 93(2): 243-7.

39. Pinzon JB, Serrano NC, Diaz LA, Mantilla G, Velasco HM, Martinez LX, et al. [Impact of the new definitions in the prevalence of the metabolic syndrome in an adult population at Bucaramanga, Colombia]. Biomedica 2007; 27(2): 172-9.

40. Lanas F, Avezum A, Bautista LE, Diaz R, Luna M, Islam S, et al. Risk factors for acute myocardial infarction in Latin America: the INTERHEART Latin American study. Circulation 2007; 115(9): 1067-74. 